\title{
Article \\ Robust Silica-Bacterial Cellulose Composite Aerogel Fibers for Thermal Insulation Textile
}

\author{
Huazheng Sai ${ }^{1,2,3, *(\mathbb{D}}$, Meijuan Wang 1,2,3, Changqing Miao 1,2,3, Qiqi Song 1,2,3, Yutong Wang 1,2,3, Rui Fu 1,2,3,*, \\ Yaxiong Wang ${ }^{1,2,3}$, Litong Ma ${ }^{1,2,3}$ and Yan Hao ${ }^{1,2,3}$
}

1 School of Chemistry and Chemical Engineering, Inner Mongolia University of Science \& Technology, Baotou 014010, China; wmjbest1014@163.com (M.W.); qingmc@163.com (C.M.); songqiqiaa@163.com (Q.S.); wangyut@163.com (Y.W.); wangyaxiong2021@126.com (Y.W.); mlt0916@126.com (L.M.); haoyannk@163.com (Y.H.)

2 Inner Mongolia Engineering Research Center of Comprehensive Utilization of Bio-Coal Chemical Industry, Inner Mongolia University of Science \& Technology, Baotou 014010, China

3 Inner Mongolia Key Laboratory of Coal Chemical Engineering \& Comprehensive Utilization, Inner Mongolia University of Science \& Technology, Baotou 014010, China

* Correspondence: shz15@tsinghua.org.cn (H.S.); furui14@mails.ucas.edu.cn (R.F.)

Citation: Sai, H.; Wang, M.; Miao, C.; Song, Q.; Wang, Y.; Fu, R.; Wang, Y.; Ma, L.; Hao, Y. Robust Silica-Bacterial Cellulose Composite Aerogel Fibers for Thermal Insulation Textile. Gels 2021, 7, 145. https://doi.org/ $10.3390 /$ gels7030145

Academic Editors: István Lázár and Melita Menelaou

Received: 13 August 2021

Accepted: 14 September 2021

Published: 17 September 2021

Publisher's Note: MDPI stays neutral with regard to jurisdictional claims in published maps and institutional affiliations.

Copyright: (c) 2021 by the authors. Licensee MDPI, Basel, Switzerland. This article is an open access article distributed under the terms and conditions of the Creative Commons Attribution (CC BY) license (https:// creativecommons.org/licenses/by/ $4.0 /)$.

\begin{abstract}
Aerogels are nanoporous materials with excellent properties, especially super thermal insulation. However, owing to their serious high brittleness, the macroscopic forms of aerogels are not sufficiently rich for the application in some fields, such as thermal insulation clothing fabric. Recently, freeze spinning and wet spinning have been attempted for the synthesis of aerogel fibers. In this study, robust fibrous silica-bacterial cellulose (BC) composite aerogels with high performance were synthesized in a novel way. Silica sol was diffused into a fiber-like matrix, which was obtained by cutting the $\mathrm{BC}$ hydrogel and followed by secondary shaping to form a composite wet gel fiber with a nanoscale interpenetrating network structure. The tensile strength of the resulting aerogel fibers reached up to $5.4 \mathrm{MPa}$ because the quantity of $\mathrm{BC}$ nanofibers in the unit volume of the matrix was improved significantly by the secondary shaping process. In addition, the composite aerogel fibers had a high specific area (up to $606.9 \mathrm{~m}^{2} / \mathrm{g}$ ), low density (less than $0.164 \mathrm{~g} / \mathrm{cm}^{3}$ ), and outstanding hydrophobicity. Most notably, they exhibited excellent thermal insulation performance in hightemperature $\left(210{ }^{\circ} \mathrm{C}\right)$ or low-temperature $\left(-72{ }^{\circ} \mathrm{C}\right)$ environments. Moreover, the thermal stability of CAFs (decomposition temperature was about $330^{\circ} \mathrm{C}$ ) was higher than that of natural polymer fiber. A novel method was proposed herein to prepare aerogel fibers with excellent performance to meet the requirements of wearable applications.
\end{abstract}

Keywords: fibrous aerogel; nanoscale interpenetrating network; secondary shaping; strength; thermal properties

\section{Introduction}

Aerogels are materials with excellent features, such as large specific surface area (500-1200 $\left.\mathrm{m}^{2} / \mathrm{g}\right)$, high porosity (80-99.8\%), and low density $\left(0.003-0.5 \mathrm{~g} / \mathrm{cm}^{3}\right)$ [1], which make them readily applicable in adsorption [2,3], heat preservation $[4,5]$, and catalysis [6,7]. However, as ultra-porous materials, aerogels are often highly brittle, especially in the case of a three-dimensional gel skeleton composed of nanoparticles, e.g., silica and other inorganic oxide aerogels [8]. This poor mechanical property is mainly due to the very small connection area between the nanoparticles that make up the gel skeleton [9]. Hence, the utilization and promotion of aerogels are severely restricted by their low mechanical strength.

Researchers worldwide have mainly focused on improving the mechanical properties of aerogel materials through precursor regulation and external doping. Methods of regulating gel precursors include increasing the quantity of precursors [10], using precursors 
containing inert groups [11-13], using precursors with flexible chains [14-16], and using precursors containing polymer monomers to construct bimolecular chain cross-linking network structures $[17,18]$ to reduce the content of rigid $-\mathrm{Si}-\mathrm{O}-\mathrm{Si}-$, giving the gel skeleton excellent flexibility to resist the impact of external forces. The external doping strategy includes a mixed extrusion of aerogel powder and long fiber [19], dispersion fiber doping [20], reinforcement by fiber felt $[21,22]$ and isometric growth of polymers on the aerogel skeleton $[23,24]$ to connect different areas of the skeleton or expend the connection area of the nanoparticles. These two strategies improve the mechanical properties to a great extent, but they are mainly applied to aerogel blocks, sheets, and blankets.

At present, the excellent thermal insulation performance of aerogel materials makes them suitable for application in thermal insulation clothing and other fabrics, which would require the processing and manufacture of aerogels as high-strength fibers. Aerogel fibers not only enrich the morphology of aerogels but also expand the applicability of aerogels in different fields and contexts. In addition to thermal insulation applications $[25,26]$, aerogel fibers could also be used in other fields, such as adsorption [27-29], biological sensing [30], and supercapacitors [31]. As a result of these potential advantages, it is essential to develop different strategies to obtain high-strength aerogel fibers.

Freeze spinning and wet spinning are the most commonly reported methods used to prepare aerogel fibers [32]. The raw materials of the aerogel fibers are typically synthetic polymers [33], natural polymers [34], graphene oxide [35], or their composites [36]. Although some studies have reported on inorganic oxide (e.g., $\mathrm{SiO}_{2}$ ) aerogels, their tensile strength generally does not exceed $0.5 \mathrm{MPa}[27,37]$, which is much lower than that of the previously mentioned fibrous aerogels. This is because there are significant differences between their microstructures. As a result of the extremely limited particle connection area, the "pearl-necklace" network formed by inorganic oxide nanoparticles is weaker than the gel skeleton composite of nanofiber-like or nanosheet-like nanostructure units connected to each other [38]. Unlike direct freeze spinning or wet spinning, a hollow polymer fiber is prepared by coaxial wet spinning, and then silk fibroin, graphene oxide, etc. are poured into it. Subsequently, the fiber being freeze dried to construct a gel skeleton was achieved recently $[26,36]$. The tensile strength of the obtained sheath-core coaxial aerogel fibers is significantly improved compared with that of fibrous aerogels without an outer polymer layer. This preparation process, which uses coaxial wet spinning to construct the hollow polymer fiber as a protective layer, is complicated and time consuming. Therefore, it is critical to continuously explore new strategies to prepare high-strength aerogel fibers, especially inorganic oxide-based aerogel fibers with excellent thermal insulation properties.

In this paper, a novel method that does not use spinning is proposed for fabricating aerogel fibers with a nanoscale interpenetrating network (as shown in Figure 1), which is a structure that has been proven to significantly improve the mechanical properties of aerogel blocks or films. Bacterial cellulose (BC) hydrogel was processed into a long fiber as the matrix, and then, the silica sol was diffused into the matrix. After secondary shaping to regulate the matrix morphology at both macroscopic and microscopic levels, the nanoscale interpenetrating network structure was obtained by an in situ sol-gel reaction. Finally, silica-BC composite aerogel fibers (CAFs) were obtained by hydrophobic modification and atmospheric pressure drying. The secondary shaping process, which increases the content of $\mathrm{BC}$ nanofibers per unit volume of the matrix, combined with the excellent mechanical properties of BC [39], significantly improves the tensile strength of the silica aerogel fibers. 


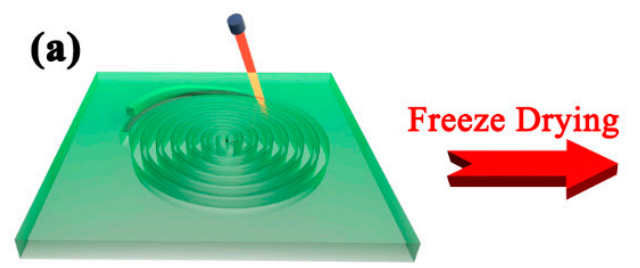

BC Hydrogel Cut by Laser (b)

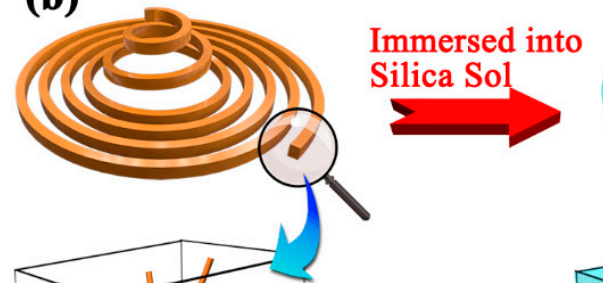

(c)

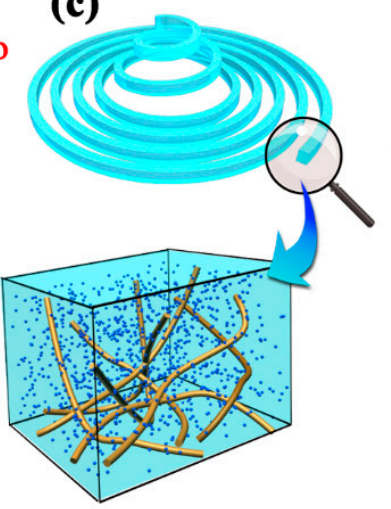

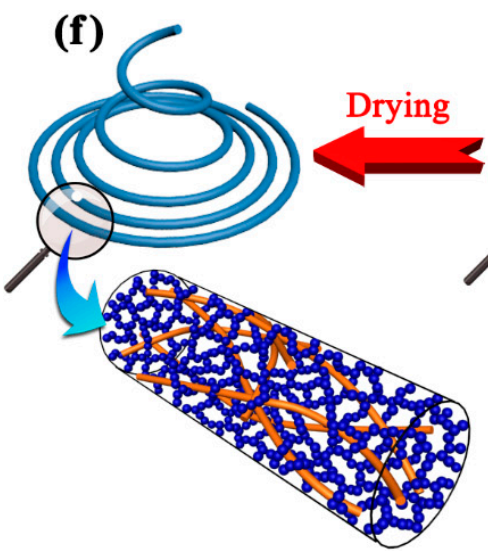

(e)

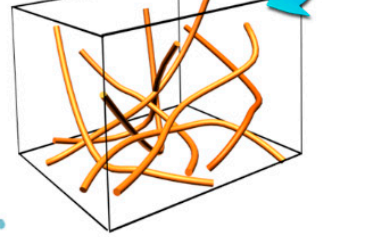

(d)

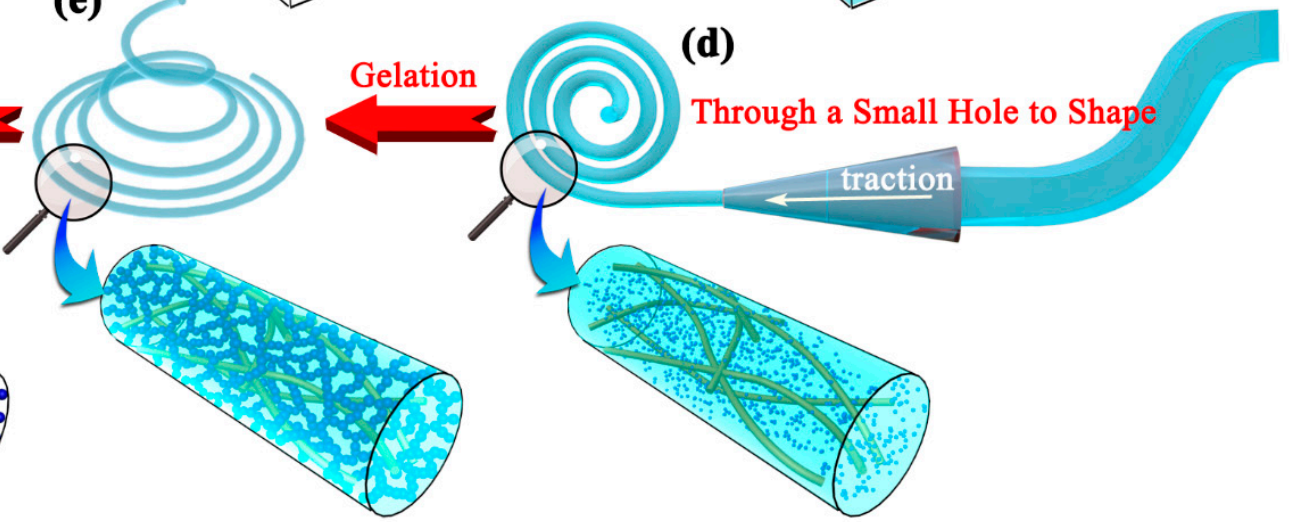

Figure 1. Schematic of the preparation process of CAFs. A nata-de-coco slice was cut by a laser (a), followed by freeze drying to obtain a fiber-like matrix consisting of BC nanofibers (b). The matrix containing silica sols (c) was reshaped by a small hole mold (d). After the silica gel skeleton was formed in the matrix (e), the fiber-like composite wet gel was dried at ambient pressure after hydrophobization to obtain CAFs (f).

\section{Results and Discussion}

\subsection{Synthesis of CAFs and Their Macroscopic Characteristics}

The detailed preparation method was described in the section "Materials and Methods part (Section 4). The diameter of the obtained CAFs was approximately $0.7 \mathrm{~mm}$, as obtained from scanning electron microscopy (SEM) images; this diameter was close to the inner diameter of the mold. This means that the sample was able to almost completely spring back in the process of atmospheric drying owing to the effective hydrophobic modification. The hydroxyl groups (including $-\mathrm{C}-\mathrm{OH}$ and $-\mathrm{Si}-\mathrm{OH}$ ) on the surface of $\mathrm{BC}$ nanofibers and silica gel skeleton were replaced with the inert and hydrophobic methyl groups through the hydrophobic modification process, which inhibited the formation of new -Si-O-Si- on the surface of the gel skeleton, allowing it to spring back during the drying process. Moreover, the hydrophobic modification also endowed the CAFs with excellent hydrophobicity. As shown in Figure 2 and Video S1, the water droplet maintained its spherical shape even when the fiber was pushed against the droplet. The excellent hydrophobicity could prevent the nanoporous structure of the CAFs from being destroyed by water vapor or water droplets. 


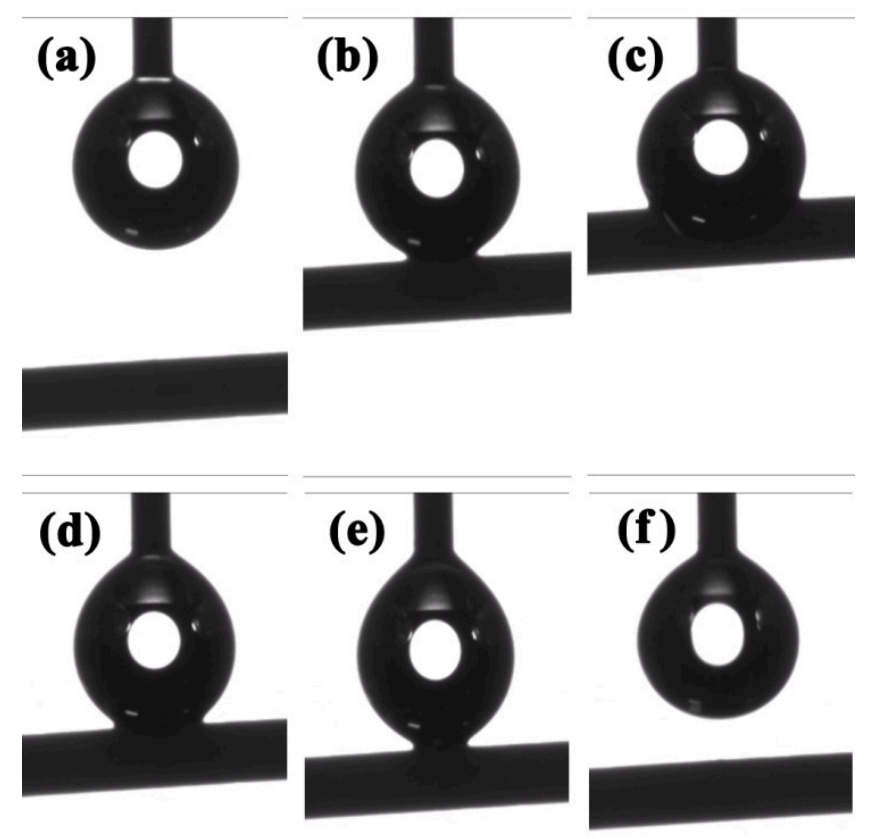

Figure 2. Wettability of CAF-3. $(\mathbf{a}-\mathbf{c})$ The gradual contact process between water droplets and the CAF. (d-f) The process of water droplets leaving the CAF.

\subsection{Microstructures}

As shown in the SEM images (Figure 3), when the precursor concentration was low, the CAF-1 was mainly composed of BC nanofiber aggregates, with only a small amount of silica nanoparticles attached to the $\mathrm{BC}$ by hydrogen bonding. At larger precursor concentrations, as in CAF-2 and CAF-3, the silica gel skeleton was gradually formed in the BC nanofiber network. Thus, the nanoscale interpenetrating network structure was constructed. When the precursor concentration was improved further, the silica gel skeleton became more compact, as shown in CAF-4. It can be seen from the cross-section that the diameter of the sample is approximately $0.7 \mathrm{~mm}$, which is close to the inner diameter of the secondary shaping mold, indicating that the sample did not significant shrink during the atmospheric pressure drying process. The diameter of CAF-1 was slightly smaller than those of the other samples because of the lack of a rigid silica gel skeleton, which caused the sample to shrink by a certain extent during the drying process. As the gel skeleton first shrinks and then springs back during the drying process, the gel skeleton must reach a certain strength to ensure that it can effectively spring back. As a result, a certain concentration of precursors (TEOS) to construct a gel skeleton with sufficient strength is very important for the preparation of composite aerogel fibers.

In addition, the spatial distribution of BC nanofibers in CAFs was obviously denser than that of $B C$ nanofibers without secondary shaping (Figure S2), which is beneficial for further improving the strength of the composite aerogels. Compared with our previous study, the higher density of the obtained BC nanofibers enables the samples to better retain their morphology without shrinkage, even at low concentrations of silica precursors [40]. This could be because denser BC nanofibers could more effectively resist the shrinkage caused by capillary forces during the ambient pressure drying process.

The nitrogen adsorption-desorption isotherms of the prepared CAFs (Figure 4a) were type IV isotherms with hysteresis loops, confirming the formation of mesoporous structures. The hysteresis loops of CAF- 3 and CAF- 4 were more obvious than those of CAF- 1 and CAF-2. Moreover, the pore size distribution (Figure $4 \mathrm{~b}$ ) showed that CAF-3 and CAF-4 had more significant mesoporous structure characteristics than CAF-1 and CAF-2. This 
suggests that sufficient silica precursors are required to form a complete gel skeleton. When the concentration of silica precursor was relatively low, there was a lack of sufficient silica nanoparticles among the nanofiber network to form a gel skeleton due to the attachment of silica nanoparticles to $\mathrm{BC}$ nanofibers. In particular, the number of $\mathrm{BC}$ nanofibers per unit volume was enhanced by secondary shaping, which further enhanced the adhesion of silica particles to the BC nanofibers. Based on the results of a BET test, the specific surface areas of the samples grew from 367.9 to $606.9 \mathrm{~m}^{2} / \mathrm{g}$ (Table 1) with an increase in TEOS concentration, which is higher than that of PAO@ANF symbiotic aerogel fiber [28] and Kevlar aerogel fibers [25]. This is because a gel skeleton composed of inorganic oxide nanoparticles has a rougher surface compared to a gel skeleton composed of nanofibers or nanosheets.

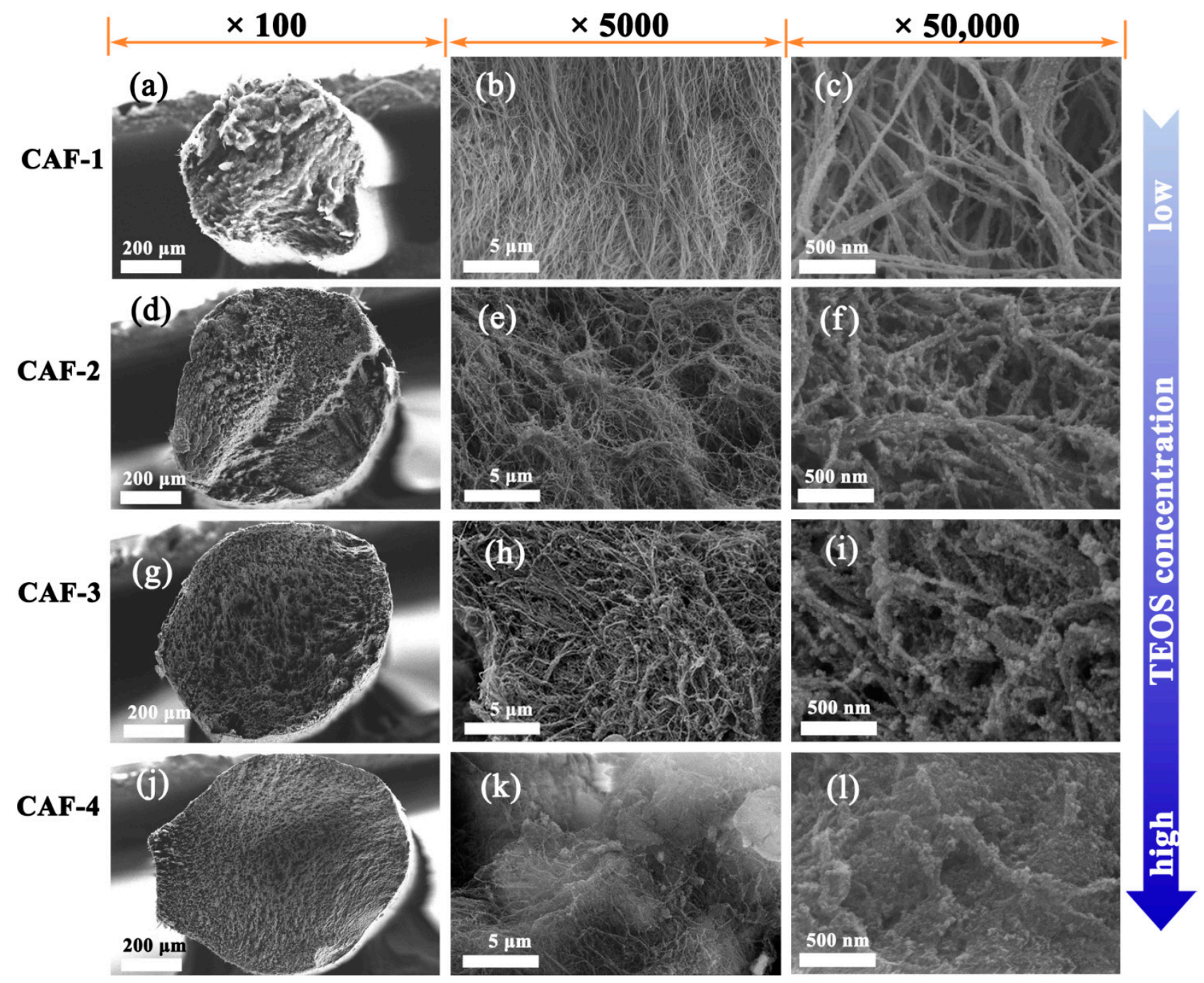

Figure 3. The SEM images of CAF-1 (a-c), CAF-2 (d-f), CAF-3 (g-i), and CAF-4 (j-1) with different magnifications. 

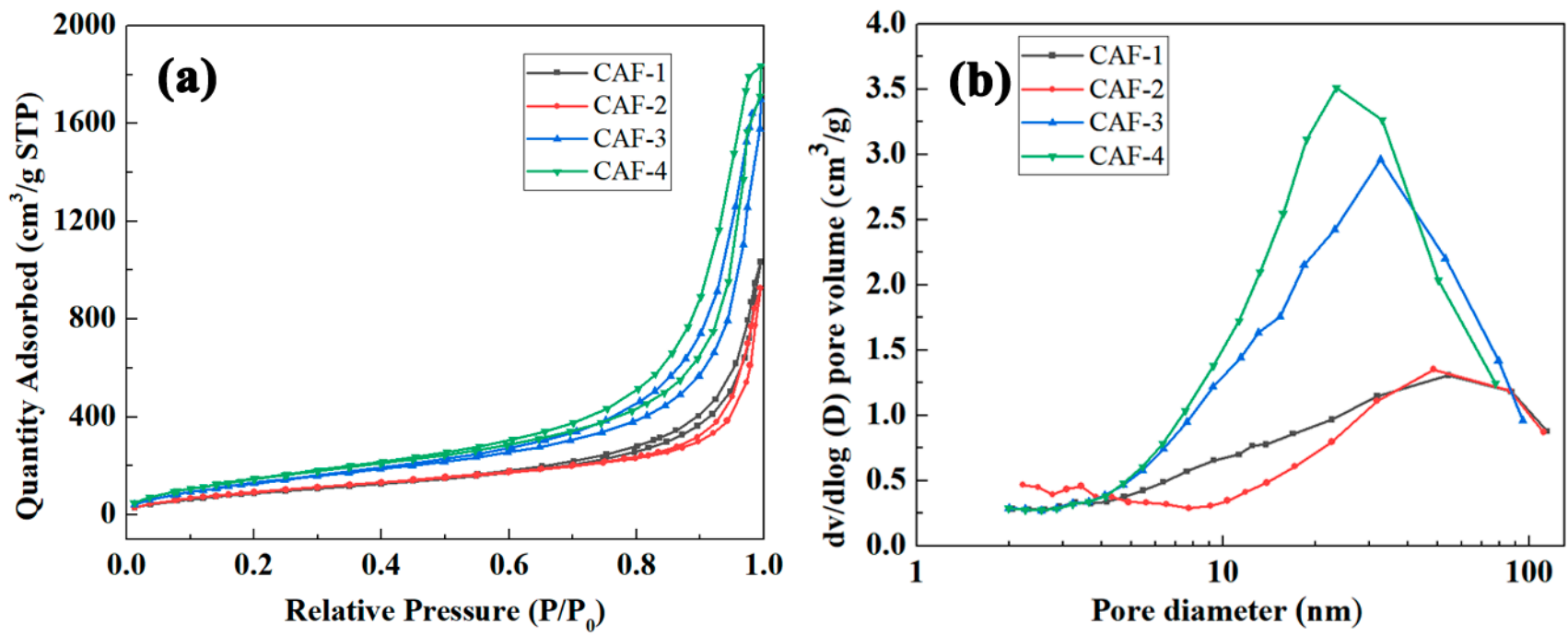

Figure 4. Nitrogen adsorption-desorption isotherms (a) and pore size distribution (b) of CAFs.

Table 1. Physical properties of CAFs.

\begin{tabular}{cccccc}
\hline Samples & $\begin{array}{c}\mathrm{SiO}_{\mathbf{2}} \text { in Aerogels } \\
{[\% \text { w/w }]}\end{array}$ & $\begin{array}{c}\text { Bulk Density } \\
{\left[\mathbf{g ~ c m}^{-3}\right]}\end{array}$ & $\begin{array}{c}\mathbf{S}_{\text {BET }} \\
{\left[\mathbf{m}^{\mathbf{2}} \mathbf{g}^{-\mathbf{1}}\right]}\end{array}$ & Pore Size [nm] & $\begin{array}{c}\text { Porosity } \\
{[\%]}\end{array}$ \\
\hline CAF-1 & 27 & 0.110 & 367.9 & 14.2 & 93.6 \\
CAF-2 & 40 & 0.121 & 387.6 & 13.7 & 93.3 \\
CAF-3 & 49 & 0.143 & 541.1 & 15.5 & 92.2 \\
CAF-4 & 55 & 0.164 & 606.9 & 15.1 & 91.2 \\
\hline
\end{tabular}

a The porosity includes the voids caused by crystal growth among gel skeletons during gel freezing.

\subsection{Mechanical Properties}

High mechanical performance is crucial for a wearable thermal insulation material. The mechanical performances of CAFs prepared with different concentrations of TEOS precursor are presented in Figure 5. The stress-strain curves of CAFs obtained from tensile tests showed that the breaking stress was in the range of 4.5-5.4 MPa. All the samples showed good tensile strength, which was much higher than that of native silica aerogel fibers, and it was comparable or even superior to those of CA/PAA-SF aerogel fibers [41] (3 MPa), SF/GO aerogel fibers [36] (3.2 MPa), and PAO@ANF aerogel fibers [28] (4.56 MPa). Moreover, the curves also showed that the elongation at break of the sample decreased from $6.8 \%$ to $1.1 \%$ with increasing precursor concentration. This could be because when more and denser gel skeletons formed between the $\mathrm{BC}$ nanofibers, the free movement of the nanofibers was restricted, resulting in the free deformation space of the nanofiber network being compressed. At the same time, the sample density increased slightly with increasing precursor concentration because of the enhancement of silica that forms the gel skeleton in the obtained CAFs, but it was generally in a low range (Table 1). 


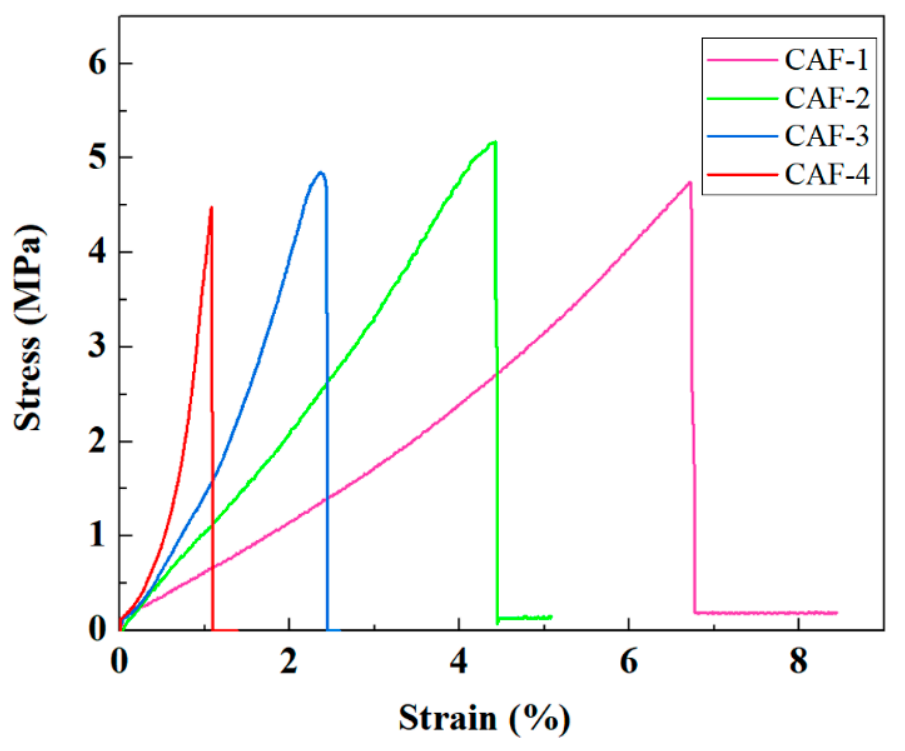

Figure 5. Stress-strain curves of CAFs.

\subsection{Thermal Insulation}

The insulation performance of CAFs obtained from different concentrations of TEOS was tested under hot and cold conditions. Cotton threads and silk fabric, with similar diameters or thicknesses as those of the CAFs, were also tested under the same conditions. First, several CAFs were packed tightly and aligned in one direction to form a single-layer mat with a thickness of approximately $0.7 \mathrm{~mm}$ and placed on a hot plate. The thermocouple was connected to the fiber surface and the hot plate, and the temperature of the surface of CAFs $\left(T_{f}\right)$ was recorded during the heating of the hot plate from 30 to $200{ }^{\circ} \mathrm{C}$. The absolute value of the temperature difference $(|\Delta T|)$ of the CAF surface $\left(T_{f}\right)$ and the hot plate were plotted against the hot plate temperature $\left(T_{h}\right)$. As shown in Figure 6a, while heating the hot plate from 30 to $200{ }^{\circ} \mathrm{C}$, the temperature difference of the one-layer CAF mat was always higher than those of the one-layer silk fabric mat and cotton fabric mat. When $T_{h}$ was $120^{\circ} \mathrm{C}$, the CAF mat temperature reached $79^{\circ} \mathrm{C}$, and the temperatures of the silk and cotton fabric mats reached $101.3^{\circ} \mathrm{C}$ and $107^{\circ} \mathrm{C}$, respectively. The higher the temperature difference $(|\Delta T|)$, the better the thermal insulation of the studied materials. Hence, the thermal insulation properties of CAFs are superior to those of silk fabrics and cotton threads. Meanwhile, for visually comparing the thermal insulation difference between the CAF fabric and cotton threads at the same heat source temperature $\left(80^{\circ} \mathrm{C}\right)$, a layer of CAF-3 formed by approximately 10 samples and a layer of 10 cotton threads of the same length were heated at the same time. After the temperature was stable, an infrared camera was used to capture photographs (Figure 6b), which showed that the temperature of the cotton fabric was higher than that of the CAF fabric, intuitively proving that the CAFs have excellent thermal insulation properties.

As seen in Figure 6a, with an increase in the amounts of TEOS in CAF-1, CAF-2, and CAF-3, the thermal insulation performances of the corresponding CAFs were gradually enhanced, owing to the complete gel skeleton gradually forming with increasing solid concentration in the aerogel. However, when the concentration of the silica precursor increased further (CAF-4), the thermal insulation performance of CAF-4 decreased because of the high content of the solid phase and because heat is more easily transmitted within the solid phase. At the same time, when the temperature was high, the slope of $|\Delta T|$ to $T_{h}$ decreased slightly with an increase in temperature, implying that the proportion of thermal radiation on heat transfer rose gradually at high temperatures. In fact, the thermal insulation mechanism of aerogels mainly depended on the blocking of thermal convection and heat conduction, not thermal radiation. Figure $6 \mathrm{c}$ shows the precise values of temperature change on the surface of the hot plate $\left(T_{h}\right)$ and aerogel fibers (CAF-3) during 
the heating process of the hot plate. The surface temperature of CAF-3 varied from 28 to $131{ }^{\circ} \mathrm{C}$ as the temperature of the hot plate rose from 30 to $210{ }^{\circ} \mathrm{C}$. When $T_{h}$ was stable at $210{ }^{\circ} \mathrm{C}$, the $|\Delta T|$ of the hot plate and CAF-3 was approximately $80^{\circ} \mathrm{C}$. When CAF-3 was heated again after a heating-cooling process, the $|\Delta T|$ showed no obvious change, indicating that the thermal insulation performance of CAF-3 was stable. The temperaturetime curves for the other samples are shown in Figure S3 (in the Supporting Information) and indicate the stability of the thermal insulation.
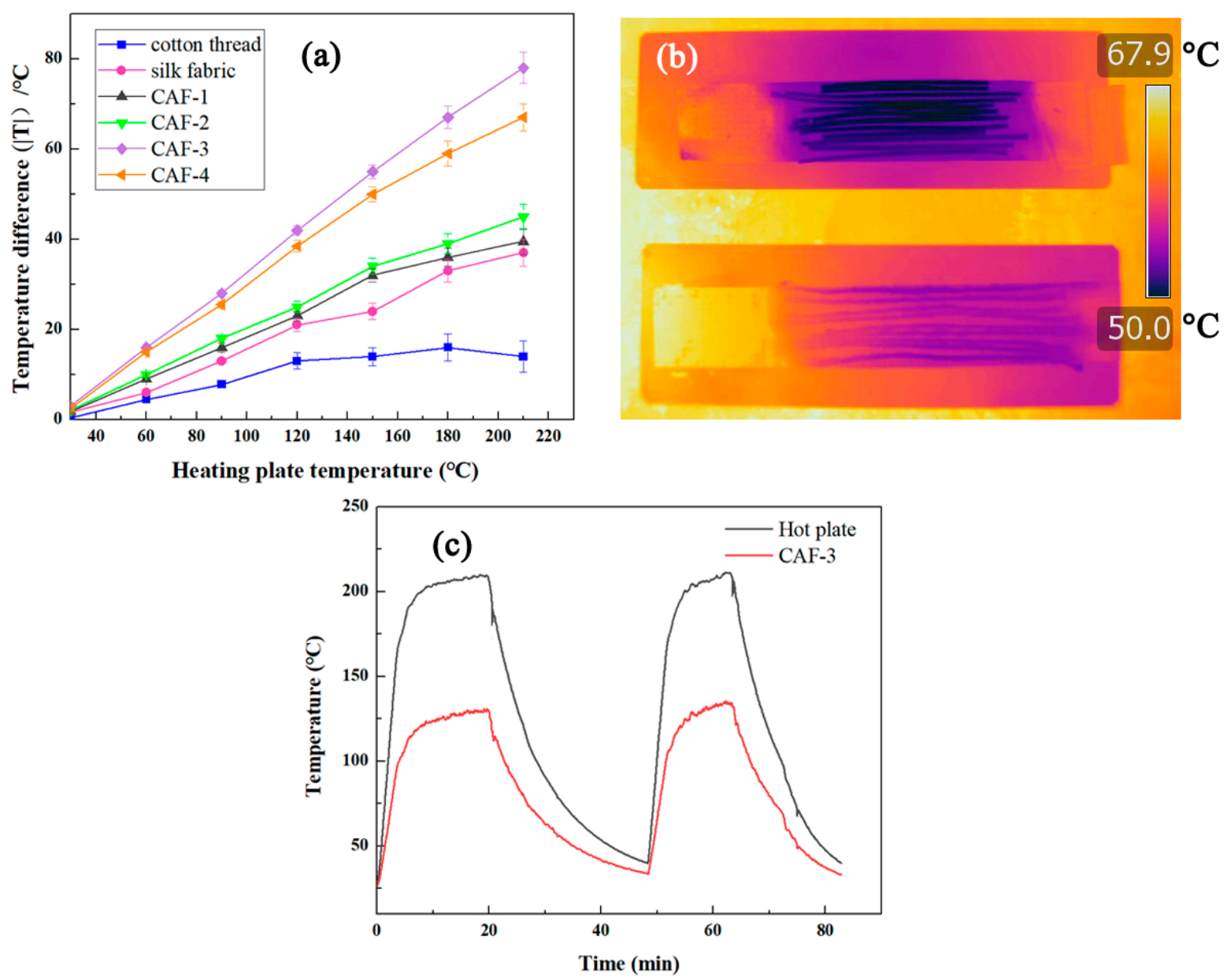

Figure 6. Thermal insulation properties of CAFs, silk fabric, and cotton threads. Temperature difference between the fiber surface and hot plate versus the hot plate for the single-layer mat of CAFs, silk fabric, and cotton threads (a). Infrared photo of one-layer mats of CAF-3 and cotton threads at high temperatures (b). Temperature-time curves of CAF-3 and hot plate (c).

To test the thermal insulation performance of the sample at lower temperatures, the three single-layer fiber mats of CAF-3, silk fabric, and cotton threads were placed on a sheet of iron with $2.5 \mathrm{~cm}$ of dry ice underneath. The temperatures of the fiber surface and iron sheet were monitored simultaneously. When the sheet temperature was $-72{ }^{\circ} \mathrm{C}$, the absolute temperature difference $|\Delta T|$ values of the one-layer aerogel fiber mat, silk fabric mat, and cotton thread mat were $59^{\circ} \mathrm{C}, 22^{\circ} \mathrm{C}$, and $46^{\circ} \mathrm{C}$, respectively. This demonstrates that CAF-3 has excellent insulation performance in cold environments.

\subsection{Thermal Stability}

As shown in Figure 7a, the BC matrix demonstrates thermal stability similar to that of cotton thread and silk fabric, which are also composites of natural polymers. The thermogravimetric analysis curves showed that the temperature of decomposition of the cellulose (BC matrix) in composite aerogel fibers gradually shifted from about $280{ }^{\circ} \mathrm{C}$ 
to about $330{ }^{\circ} \mathrm{C}$ (Figure $7 \mathrm{~b}$ ) as the silica content increased. Moreover, when the mass gradually decreased to $88 \%$ of the initial mass, as shown by the blue arrow in Figure $7 \mathrm{~b}$, the temperature corresponding to the sample gradually rose from $300{ }^{\circ} \mathrm{C}$ to about $370{ }^{\circ} \mathrm{C}$ as the silica content increased. Hence, silica improved the thermal stability of the polymer matrix. This allows the CAFs to be used at higher temperatures than the traditional polymer fiber, owing to the stabilizing effect of silica on $\mathrm{BC}$ matrix.
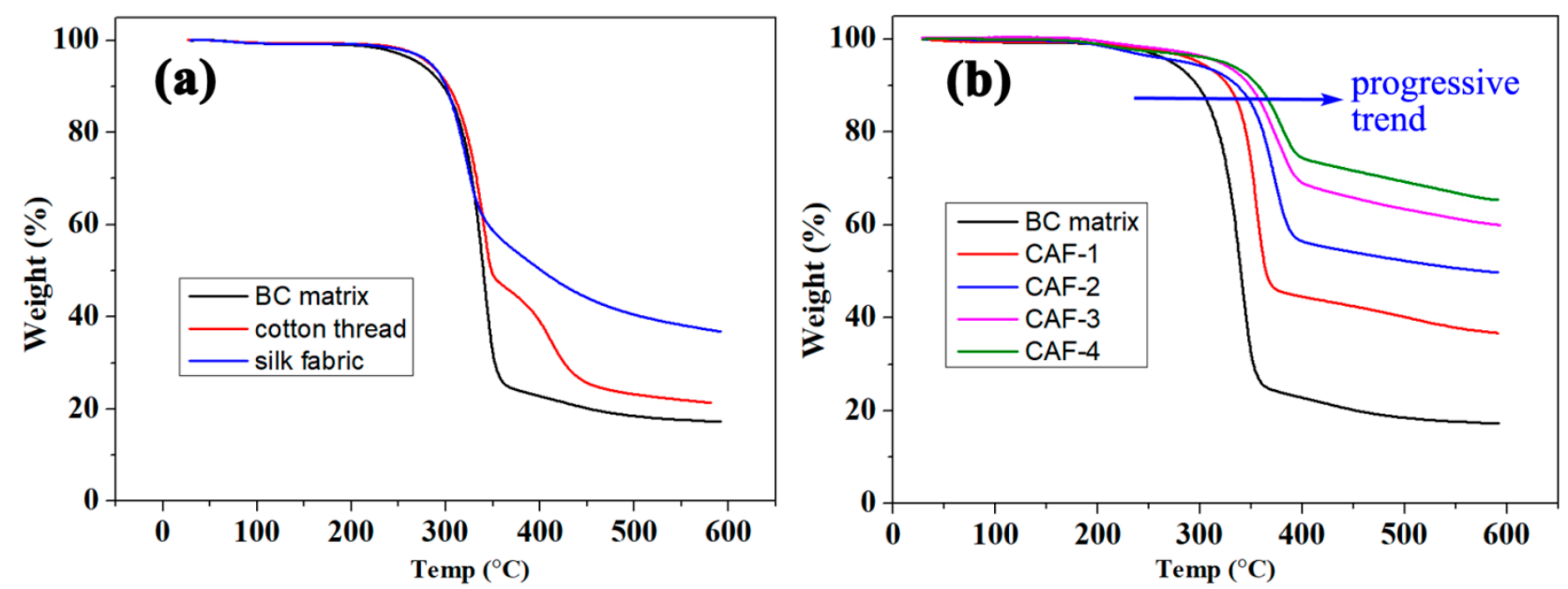

Figure 7. (a) Thermogravimetry analysis (TGA, $10^{\circ} \mathrm{C} \mathrm{min}{ }^{-1}$ heating) curves of $\mathrm{BC}$ matrix, cotton thread, and silk fabric. (b) Thermogravimetry analysis (TGA, $10{ }^{\circ} \mathrm{C} \mathrm{min}^{-1}$ heating) curves of $\mathrm{BC}$ matrix and CAFs. The blue arrow shows the temperature changes of each material at the same change in weight proportion $(88 \%)$.

\section{Conclusions}

In summary, a novel and simple method for preparing aerogel fibers with excellent mechanical properties and thermal insulation has been demonstrated here. The silica precursor TEOS and BC matrix were used to obtain the CAFs through in situ sol-gel reaction. The mechanical properties of CAFs were significantly improved by increasing the content of $\mathrm{BC}$ nanofibers per unit volume via a secondary shaping process. Efficient silica gel skeleton formed in the $\mathrm{BC}$ matrix endowed the CAFs with excellent thermal insulation performance and large specific surface area $\left(606.9 \mathrm{~m}^{2} / \mathrm{g}\right)$. Moreover, the stabilizing effect of silica on the $\mathrm{BC}$ matrix makes the CAFs usable at relatively high temperatures (up to about $330^{\circ} \mathrm{C}$ ). In addition, the outstanding hydrophobicity of CAFs enables them to resist erosion by water vapor and have good weather resistance. Consequently, these aerogel fibers are promising materials for wearable thermal insulation. This work introduced the nanoscale interpenetrating network structure into aerogel fibers and provided a new way for the preparation and toughening of aerogel fibers, especially the inorganic oxide-based aerogel fibers.

\section{Materials and Methods}

\subsection{Materials}

Nata-de-coco slices were purchased from Wenchang Baocheng Industry and Trade Co., Ltd. (Hainan, China). Tetraethoxysilane (TEOS), n-hexane, triethylamine (TEA), trimethylchlorosilane (TMCS), and tert-butanol were obtained from Aladdin Reagent Co., Ltd. (Shanghai, China). Ethanol, hydrochloric acid $(\mathrm{HCl})$, sodium hydroxide $(\mathrm{NaOH}$, $4 \mathrm{wt} \%)$, and ammonium hydroxide $\left(\mathrm{NH}_{3} \cdot \mathrm{H}_{2} \mathrm{O}\right)$ were purchased from Beijing Chemical Reagent Co. (Beijing, China), LTD. All the chemicals were of analytical grade and were used as received without any further purification.

\subsection{Preparation of $B C$ Fibers}

Nata-de-coco slices (i.e., bacterial cellulose hydrogel, BC hydrogel) of thicknesses of $3 \mathrm{~mm}$ were first soaked in deionized water for $4 \mathrm{~h}$. The water was replaced several times to 
Nata-de-coco slice i.e. BC hydrogel

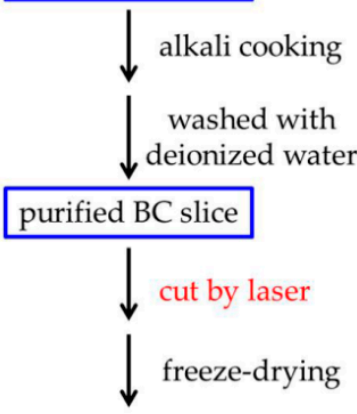

dried fiber-like BC matrix remove the added sucrose; after this process, the slices were boiled in $\mathrm{NaOH}$ for $6 \mathrm{~h}$ at $90^{\circ} \mathrm{C}$. Subsequently, clean $\mathrm{BC}$ hydrogel was obtained by rinsing it with deionized water until it became neutral. The nata-de-coco slice (Figure S1a) was placed on a glass plate, squeezed to remove about $80 \%$ of the water, and then cut using a laser $(15 \mathrm{~W}$ power, $80 \mathrm{~W} 6040$, Liaocheng Julong Laser Equipment Co., Ltd., Liaocheng, China) to obtain fibers of uniform width $(2 \mathrm{~mm}$ ) and length (about $500 \mathrm{~mm}$ ) (Figure 1a and Figure S1b). Finally, the dried fiberlike BC matrix (Figure $1 \mathrm{~b}$ and Figure S1c) was obtained by freeze drying for $24 \mathrm{~h}$ after the solvent replacement of the mixed liquid of water and tert-butanol $\left(\mathrm{V}_{\text {water }}: \mathrm{V}_{\text {tert-butanol }}=3: 2\right)$. This step was shown on the left side of Figure 8. 


\subsection{Hydrophobic Modification and Atmospheric Drying of CAFs}

The wet gel fibers were soaked in ethanol and heated at $70{ }^{\circ} \mathrm{C}$ for $1 \mathrm{~h}$ to age the silica gel skeleton. Then, ethanol was replaced with $n$-hexane for $3 \mathrm{~h}$ for solvent replacement. $\mathrm{N}$-hexane $(50 \mathrm{~mL})$, TEA $(4 \mathrm{~mL})$, and TMCS $(3 \mathrm{~mL})$ were added to the glass reactor, and the wet gel fibers were immersed in the liquid mixture. The glass reactor was heated in an oil bath and refluxed for $2 \mathrm{~h}$. Then, the wet gel fibers were dipped into a $100 \mathrm{~mL}$ beaker containing $50 \mathrm{~mL}$ ethanol, which was replaced every $30 \mathrm{~min}$; this process was repeated twice. Subsequently, ethanol was replaced with $n$-hexane, and the same operation as above was repeated. Subsequently, the wet gel fibers after hydrophobic modification were removed and heated in an oven at $80^{\circ} \mathrm{C}$ for $20 \mathrm{~min}$. Finally, the hydrophobic CAFs were obtained (Figure $1 \mathrm{f}$ and Figure S1f).

\subsection{Characterization}

As shown in the upper right corner of Figure 8, the morphology of the CAFs was determined, and the specific surface area, porosity, pore-size distribution, mechanical properties, wettability, density, and content of silica in the CAFs were examined. Thermal insulation performance and thermal stability were also evaluated. Detailed characterization methods are provided in the Supporting Information.

Supplementary Materials: The following are available online at https:/ /www.mdpi.com/article/10 .3390 /gels7030145/s1, Video S1: video of the wettability of the silica-bacterial cellulose composite aerogel fibers (CAFs), Figure S1: Photos of the preparation process of CAFs; Figure S2: SEM images of BC without secondary shaping; Figure S3: Temperature-time curves of CAFs at high temperature; Characterization and Reference.

Author Contributions: Conceptualization, project administration, validation, funding acquisition, writing-review and editing, H.S.; investigation, data curation, writing-original draft, M.W.; methodology, C.M.; validation, Q.S.; data curation, Y.W. (Yutong Wang); supervision, conceptualization, R.F.; project administration, Y.W. (Yaxiong Wang); supervision, L.M.; funding acquisition, Y.H. All authors have read and agreed to the published version of the manuscript.

Funding: This research was funded by the Innovation Fund of Inner Mongolia University of Science \& Technology (2018QDL-B01), the Natural Science Foundation of Inner Mongolia (2019BS05022, 2019LH02004), the Scientific Research Projects of Colleges and Universities of Inner Mongolia (NJZZ20087), Science and Technology Plan Project of Inner Mongolia Autonomous Region (2020GG0152), National Natural Science Foundation of China (NNSFC, No. 52164013 and No. 11965015) and Outstanding Youth Fund Project of Innovation Fund of Inner Mongolia University of Science and Technology (No. 2019YQL05). We also appreciate the financial support from Human Resources and Social Security Department of Inner Mongolia.

Institutional Review Board Statement: Not applicable.

Informed Consent Statement: Not applicable.

Conflicts of Interest: The authors declare no conflict of interest.

\section{References}

1. Ziegler, C.; Wolf, A.; Liu, W.; Herrmann, A.-K.; Gaponik, N.; Eychmüller, A. Modern Inorganic Aerogels. Angeww. Chem. Int. Ed. 2017, 56, 13200-13221. [CrossRef]

2. Sai, H.; Fu, R.; Xing, L.; Xiang, J.; Li, Z.; Li, F.; Zhang, T. Surface Modification of Bacterial Cellulose Aerogels' Web-like Skeleton for Oil/Water Separation. ACS Appl. Mater. Interfaces 2015, 7, 7373-7381. [CrossRef] [PubMed]

3. Peydayesh, M.; Suter, M.K.; Bolisetty, S.; Boulos, S.; Handschin, S.; Nyström, L.; Mezzenga, R. Amyloid Fibrils Aerogel for Sustainable Removal of Organic Contaminants from Water. Adv. Mater. 2020, 32, 1907932. [CrossRef]

4. Adhikary, S.K.; Ashish, D.K.; Rudžionis, Ž. Aerogel based thermal insulating cementitious composites: A review. Energy Build. 2021, 245, 111058. [CrossRef]

5. Wu, K.; Zhang, L.; Yuan, Y.; Zhong, L.; Chen, Z.; Chi, X.; Lu, H.; Chen, Z.; Zou, R.; Li, T.; et al. An Iron-Decorated Carbon Aerogel for Rechargeable Flow and Flexible Zn-Air Batteries. Adv. Mater. 2020, 32, 2002292. [CrossRef] [PubMed]

6. Cai, B.; Eychmüller, A. Promoting Electrocatalysis upon Aerogels. Adv. Mater. 2019, 31, 1804881. [CrossRef] 
7. Fu, G.; Yan, X.; Chen, Y.; Xu, L.; Sun, D.; Lee, J.-M.; Tang, Y. Boosting Bifunctional Oxygen Electrocatalysis with 3D Graphene Aerogel-Supported Ni/MnO Particles. Adv. Mater. 2018, 30, 1704609. [CrossRef] [PubMed]

8. Adhikary, S.K.; Rudžionis, Ž.; Tučkute, S.; Ashish, D.K. Effects of carbon nanotubes on expanded glass and silica aerogel based lightweight concrete. Sci. Rep. 2021, 11, 2104. [CrossRef]

9. Mohite, D.P.; Larimore, Z.J.; Lu, H.; Mang, J.T.; Sotiriou-Leventis, C.; Leventis, N. Monolithic Hierarchical Fractal Assemblies of Silica Nanoparticles Cross-Linked with Polynorbornene via ROMP: A Structure-Property Correlation from Molecular to Bulk through Nano. Chem. Mater. 2012, 24, 3434-3448. [CrossRef]

10. Fricke, J. Aerogels-Highly tenuous solids with fascinating properties. J. Non-Cryst. Solids 1988, 100, 169-173. [CrossRef]

11. Cai, L.; Shan, G. Elastic silica aerogel using methyltrimethoxysilane precusor via ambient pressure drying. J. Porous Mat. 2015, 22, 1455-1463. [CrossRef]

12. Zhong, L.; Chen, X.; Song, H.; Guo, K.; Hu, Z. Highly flexible silica aerogels derived from methyltriethoxysilane and polydimethylsiloxane. New J. Chem. 2015, 39, 7832-7838. [CrossRef]

13. Rao, A.V.; Bhagat, S.D.; Hirashima, H.; Pajonk, G.M. Synthesis of flexible silica aerogels using methyltrimethoxysilane (MTMS) precursor. J. Colloid Interface Sci. 2006, 300, 279-285. [PubMed]

14. Wang, Z.; Dai, Z.; Wu, J.; Zhao, N.; Xu, J. Vacuum-Dried Robust Bridged Silsesquioxane Aerogels. Adv. Mater. 2013, 25, 4494-4497. [CrossRef] [PubMed]

15. Wang, Z.; Wang, D.; Qian, Z.; Guo, J.; Dong, H.; Zhao, N.; Xu, J. Robust Superhydrophobic Bridged Silsesquioxane Aerogels with Tunable Performances and Their Applications. ACS Appl. Mater. Interfaces 2015, 7, 2016-2024. [CrossRef] [PubMed]

16. Yun, S.; Luo, H.; Gao, Y. Low-density, hydrophobic, highly flexible ambient-pressure-dried monolithic bridged silsesquioxane aerogels. J. Mater. Chem. A 2015, 3, 3390-3398. [CrossRef]

17. Zu, G.; Kanamori, K.; Maeno, A.; Kaji, H.; Nakanishi, K. Superflexible Multifunctional Polyvinylpolydimethylsiloxane-Based Aerogels as Efficient Absorbents, Thermal Superinsulators, and Strain Sensors. Angew. Chem. Int. Ed. 2018, 57, 9722-9727. [CrossRef]

18. Zu, G.; Shimizu, T.; Kanamori, K.; Zhu, Y.; Maeno, A.; Kaji, H.; Shen, J.; Nakanishi, K. Transparent, Superflexible Doubly Cross-Linked Polyvinylpolymethylsiloxane Aerogel Superinsulators via Ambient Pressure Drying. ACS Nano 2018, 12, 521-532. [CrossRef]

19. Yuan, B.; Ding, S.; Wang, D.; Wang, G.; Li, H. Heat insulation properties of silica aerogel/glass fiber composites fabricated by press forming. Mater. Lett. 2012, 75, 204-206. [CrossRef]

20. Xu, L.; Jiang, Y.; Feng, J.; Feng, J.; Yue, C. Infrared-opacified $\mathrm{Al}_{2} \mathrm{O}_{3}-\mathrm{SiO}_{2}$ aerogel composites reinforced by SiC-coated mullite fibers for thermal insulations. Ceram. Int. 2015, 41, 437-442. [CrossRef]

21. Chandradass, J.; Kang, S.; Bae, D.-S. Synthesis of silica aerogel blanket by ambient drying method using water glass based precursor and glass wool modified by alumina sol. J. Non-Cryst. Solids 2008, 354, 4115-4119. [CrossRef]

22. Oh, K.; Kim, D.; Kim, S. Ultra-porous flexible PET/Aerogel blanket for sound absorption and thermal insulation. Fiber. Polym. 2009, 10, 731-737. [CrossRef]

23. Nguyen, B.N.; Meador, M.A.B.; Tousley, M.E.; Shonkwiler, B.; McCorkle, L.; Scheiman, D.A.; Palczer, A. Tailoring Elastic Properties of Silica Aerogels Cross-Linked with Polystyrene. ACS Appl. Mater. Interfaces 2009, 1, 621-630. [CrossRef] [PubMed]

24. Randall, J.P.; Meador, M.A.B.; Jana, S.C. Tailoring Mechanical Properties of Aerogels for Aerospace Applications. ACS Appl. Mater. Interfaces 2011, 3, 613-626. [CrossRef]

25. Liu, Z.; Lyu, J.; Fang, D.; Zhang, X. Nanofibrous Kevlar Aerogel Threads for Thermal Insulation in Harsh Environments. ACS Nano 2019, 13, 5703-5711. [CrossRef] [PubMed]

26. Zhou, J.; Hsieh, Y.-L. Nanocellulose aerogel-based porous coaxial fibers for thermal insulation. Nano Energy 2020, 68,104305 [CrossRef]

27. Meng, S.; Zhang, J.; Chen, W.; Wang, X.; Zhu, M. Construction of continuous hollow silica aerogel fibers with hierarchical pores and excellent adsorption performance. Microporous Mesoporous Mat. 2019, 273, 294-296. [CrossRef]

28. Li, J.; Wang, J.; Wang, W.; Zhang, X. Symbiotic Aerogel Fibers Made via In-Situ Gelation of Aramid Nanofibers with Polyamidoxime for Uranium Extraction. Molecules 2019, 24, 1821. [CrossRef]

29. Meng, S.; Zhang, J.; Xu, W.; Chen, W.; Zhu, L.; Zhou, Z.; Zhu, M. Structural control of silica aerogel fibers for methylene blue removal. Sci. China Technol. Sci. 2019, 62, 958-964. [CrossRef]

30. Mitropoulos, A.N.; Burpo, F.J.; Nguyen, C.K.; Nagelli, E.A.; Ryu, M.Y.; Wang, J.; Sims, R.K.; Woronowicz, K.; Wickiser, J.K. Noble Metal Composite Porous Silk Fibroin Aerogel Fibers. Materials 2019, 12, 894. [CrossRef]

31. Lai, H.; Wang, Y.; Wang, Y.; Liu, W.; Bao, X.; Liu, F.; Li, X.; Lei, Z.; Jiao, H.; Fan, Z. Macroscale amphiphilic aerogel fibers made from nonwoven nanofibers for large active mass loading. J. Power Sources 2020, 474, 228612. [CrossRef]

32. Liu, Y.; Zhang, Y.; Xiong, X.; Ge, P.; Wu, J.; Sun, J.; Wang, J.; Zhuo, Q.; Qin, C.; Dai, L. Strategies for Preparing Continuous Ultraflexible and Ultrastrong Poly(Vinyl Alcohol) Aerogel Fibers with Excellent Thermal Insulation. Macromol. Mater. Eng. 2021, 306, 2100399. [CrossRef]

33. Li, M.; Gan, F.; Dong, J.; Fang, Y.; Zhao, X.; Zhang, Q. Facile Preparation of Continuous and Porous Polyimide Aerogel Fibers for Multifunctional Applications. ACS Appl. Mater. Interfaces 2021, 13, 10416-10427. [CrossRef]

34. Ying, C.; Huaxin, G.; Yujie, W.; Dewen, L.; Hao, B. A Thermally Insulating Textile Inspired by Polar Bear Hair. Adv. Mater. 2018, 30, 1706807. 
35. Xu, Z.; Zhang, Y.; Li, P.; Gao, C. Strong, Conductive, Lightweight, Neat Graphene Aerogel Fibers with Aligned Pores. ACS Nano 2012, 6, 7103-7113. [CrossRef]

36. Wang, Z.; Yang, H.; Li, Y.; Zheng, X. Robust Silk Fibroin/Graphene Oxide Aerogel Fiber for Radiative Heating Textiles. ACS Appl. Mater. Interfaces 2020, 12, 15726-15736. [CrossRef]

37. Du, Y.; Zhang, X.; Wang, J.; Liu, Z.; Zhang, K.; Ji, X.; You, Y.; Zhang, X. Reaction-Spun Transparent Silica Aerogel Fibers. ACS Nano 2020, 14, 11919-11928. [CrossRef] [PubMed]

38. Zhang, G.H.; Dass, A.; Rawashdeh, A.M.M.; Thomas, J.; Counsil, J.A.; Sotiriou-Leventis, C.; Fabrizio, E.F.; Ilhan, F.; Vassilaras, P.; Scheiman, D.A.; et al. Isocyanate-crosslinked silica aerogel monoliths: Preparation and characterization. J. Non-Cryst. Solids 2004, 350, 152-164. [CrossRef]

39. Wang, S.; Jiang, F.; Xu, X.; Kuang, Y.; Fu, K.; Hitz, E.; Hu, L. Super-Strong, Super-Stiff Macrofibers with Aligned, Long Bacterial Cellulose Nanofibers. Adv. Mater. 2017, 29, 1702498. [CrossRef] [PubMed]

40. Sai, H.; Fu, R.; Xiang, J.; Guan, Y.; Zhang, F. Fabrication of elastic silica-bacterial cellulose composite aerogels with nanoscale interpenetrating network by ultrafast evaporative drying. Compos. Sci. Technol. 2018, 155, 72-80. [CrossRef]

41. Yang, H.; Wang, Z.; Liu, Z.; Cheng, H.; Li, C. Continuous, Strong, Porous Silk Firoin-Based Aerogel Fibers toward Textile Thermal Insulation. Polymers 2019, 11, 1899. [CrossRef] [PubMed] 